\title{
Betriebliches Informationsmanagement
}

\section{Thomas Mandl*, Ella Schwab, Ben Heuwing und Christa Womser-Hacker Digitalisierung in Unternehmen}

\author{
Austausch und Nutzung von Information am modernen Arbeitsplatz
}

https://doi.org/10.1515/iwp-2018-0018

Zusammenfassung: Digitale Arbeitsplätze führen zu einem tiefgehenden Wandel. Dies gilt nicht nur für technologische Neuerungen. Mit der Digitalisierung gehen Änderungen in der Unternehmens- und Kommunikationskultur einher. Diese werden in der Literatur häufig beschrieben, aber entscheidend erscheint die Perspektive der Unternehmen selbst. In welchen Situationen gelingt die Digitalisierung nicht? Welche Prozesse werden als besonders anspruchsvoll und schwierig empfunden? Wie können am schnellsten Fortschritte erzielt werden? Dieser Artikel gibt einen Überblick über die Durchführung und die Ergebnisse einer Fokusgruppe, die mit Führungskräften realisiert wurde, welche den Wandel durch Digitalisierung täglich erleben und gestalten. Sie sahen die wesentlichsten Hürden in den Bereichen Informationssysteme und Integration, Strukturen im Informationsmanagement sowie Führungskultur im Unternehmen.

Deskriptoren: Arbeitsplatz, Arbeitsplatzcomputer, Unternehmen, Digital, Betriebswirtschaft, Information, Informationsbedarf, Informationsaustausch, Informationswissenschaft, Führungskraft, Digitalisierung

\section{Digitalization in companies: Exchange and use of Information in modern work places}

Abstract: Digital work places lead to profound change in the professional life. This is not only true for technological innovations, but digitization brings with it profound

*Kontaktperson: apl. Prof. Dr. Thomas Mandl, Universität Hildesheim, Institut für Informationswissenschaft und Sprachtechnologie, Universitätsplatz 1, 31141 Hildesheim, E-Mail:mandl@uni-hildesheim.de

Ella Schwab, Universität Hildesheim, Institut für Informationswissenschaft und Sprachtechnologie, Universitätsplatz 1, 31141 Hildesheim

Dr. Ben Heuwing, ]init[ AG für digitale Kommunikation, Köpenicker Straße 9, 10997 Berlin, E-Mail: ben.heuwing@init.de Prof. Dr. Christa Womser-Hacker, Universität Hildesheim, Institut für Informationswissenschaft und Sprachtechnologie, Universitätsplatz 1, 31141 Hildesheim,

E-Mail:womser@uni-hildesheim.de changes in corporate and communication culture. These are frequently described; however, the perspective of the companies themselves seems crucial. In which situations does digitization fail? Which of the processes are perceived as particularly demanding and difficult? How can progress be achieved best? This article reports the implementation and the results of a focus group conducted with decision makers in companies who experience and shape the digitalization on a daily basis. As the most relevant obstacles they identified information systems and integration, structures in information management and leadership in companies.

Descriptors: Workplace, Workstation computers, Company, Digital, Business administration, Information, Information demand, Information exchange, Information Science, Information technology, Management, Digitisation

\section{La digitalisation dans les entreprises. Échange et utilisa- tion de l'information sur le lieu de travail moderne}

Résumé: Le numérique provoque des changements profonds sur le lieu de travail. Cela ne s'applique pas seulement aux innovations technologiques. La digitalisation s'accompagne de changements dans la culture de l'entreprise et la communication. Ceux-ci sont fréquemment décrits dans la littérature, mais la perspective des entreprises elles-mêmes semble décisive. Dans quelles situations la digitalisation échoue-t-elle ? Quels processus sont perçus comme particulièrement exigeants et difficiles ? Comment peut-t-on progresser le plus rapidement ? Cet article donne un aperçu de la mise en pratique et des résultats d'un groupe témoin réalisé avec des décideurs qui vivent et façonnent le changement dû à la digitalisation chaque jour. Ils ont vu les obstacles les plus importants dans les domaines des systèmes d'information et d'intégration, des structures de gestion de l'information et de la culture de leadership dans l'entreprise.

Descripteurs: Lieu de travail, Ordinateur personnel, Entreprise, Numérique, Gestion d'entreprise, Information, Besoin d'information, Échange d'information, Science de l'information, leadership, digitalisation 


\section{Digitalisierung von Arbeitsplätzen}

Die Digitalisierung der Arbeitswelt ist heute kein neues Phänomen mehr: Mit dem Aufkommen des World Wide Web und der zunehmenden Verbreitung von Computern in den 90er Jahren hat die Digitalisierung ihren Anfang genommen. Die rasanten Entwicklungen des vergangenen Jahrzehnts im Bereich der Informations- und Kommunikationstechnologien (IuK) haben dazu geführt, dass sich Kommunikation, Zusammenarbeit und Prozesse in der Arbeitswelt elementar verändern. Die Digitalisierung hat enorm an Dynamik gewonnen und betrifft mittlerweile jede Branche. Es gibt kaum noch Beschäftigte, die ohne Informationstechnologie tätig sind. Waren manche Technologien wie mobile Endgeräte oder Social Media zunächst als Verbraucher-Technologien einzustufen, sind sie mittlerweile auch aus Unternehmen nicht mehr wegzudenken.

Primär ergeben sich durch den technologischen Fortschritt zahlreiche Chancen, die Arbeitswelt zu modernisieren und Prozesse zu vereinfachen. Laut einer Studie der Bitkom Research sehen Unternehmen die Digitalisierung als eine der wichtigsten und größten aktuellen Herausforderungen an. Diese steht nach der Sicherung von Fachkräften für 72 Prozent von insgesamt 507 befragten Wirtschaftsunternehmen auf Platz zwei der Herausforderungen (Dirks 2016, S. 2). Auch erkennen Unternehmen die Chancen der Digitalisierung für den modernen Arbeitsplatz, aber an der erfolgreichen Realisierung scheitern viele: Ein Großteil der befragten Unternehmen (59\%) sehen sich selbst als Nachzügler und sieben Prozent befürchten gar, den Anschluss ganz verpasst zu haben (Dirks 2016, S. 5).

Im Hinblick auf die geschilderten Entwicklungen hat die europäische Arbeitsgruppe ENWI (European Network for Workplace Information) das Projekt „Digital Workplaces“ initiiert. Die Arbeitsgruppe ENWI, die sich aus etwa 20 europäischen Forschenden und Vertreterinnen ${ }^{1}$ der Industrie zusammensetzt, beschäftigt sich mit den vielschichtigen Aspekten und Herausforderungen von Informationsmanagement am Arbeitsplatz (Byström 2016; Byström et al. 2016). Ziel von ENWI ist die Unterstützung von Forschungsarbeiten in diesem Feld. Dazu entwickelt sie Konzepte, Modelle und Forschungsmethoden zur Analyse der Rolle von Information am modernen Arbeitsplatz (Widén et al. 2014). Damit bietet ENWI einen theoretischen Rahmen für die hier vorgestellte Analyse.

1 In diesem Beitrag wird zu bessern Lesbarkeit der feminine Plural für männliche und weibliche Endungen verwendet, in Einzelfällen kann aus stilistischen Gründen das Maskulinum eingesetzt werden.

\section{Stand der Forschung: Digitaler Arbeitsplatz}

Eine Definition von Arbeitsplatz muss heute mehr als Räumlichkeiten oder Kompetenzen beschreiben. White (2012, S. 209) weist den modernen Arbeitsplatz als eine virtuelle Umgebung aus: „A digital workplace enables any employee to complete a task, share information and work as a member of a team with other employees in the organization and in any partner organization on a totally location-independent basis for all the parties concerned." Diese Definition erweitert Bollier (2011, S. 7): Arbeit und Arbeitsplatz werden sich demnach nicht mehr abgrenzen lassen, sondern zu einer vagen Begrifflichkeit verschmelzen, weil Arbeit zunehmend ortsungebunden stattfindet. Der digitale Arbeitsplatz greift nicht nur auf das Territorium des „Zuhauses“ zu, sondern erstreckt sich global. Unternehmen stellen sich zunehmend als virtuelle Firmen auf und ermöglichen eine dezentrale Zusammenarbeit über Social Tools.

In Zusammenhang mit der Digitalisierung der Arbeitswelt wird oft von Industrie 4.0 gesprochen. Im englischsprachigen Raum verwendet man eher die Begriffe Industrial Internet, Integrated Industry, Smart Industry oder Smart Manufacturing, wobei sie alle ähnliche Dinge umschreiben (Herrmann et al. 2015, S. 6). Anders als die Definition des digitalen Arbeitsplatzes, bei der es darum geht, wie Beschäftigte Aufgaben innerhalb ihrer Jobrolle bewältigen, steht bei Industrie 4.0 die Transformation der industriellen Produktion im Vordergrund. Hier spielen Themen wie Robotik, Assistenzsysteme bis hin zur Künstlichen Intelligenz und das Internet der Dinge eine Rolle. Digitalisierung in der Industrie und Produktion beeinflusst auch Arbeitsplätze, allerdings primär für die in der Produktion tätigen Angestellten.

Der ortsunabhängige Zugriff auf Informationen, z. B. durch cloud-basierte IT-Infrastruktur in Kombination mit Social Tools und mobilen Endgeräten, erlaubt es den Mitarbeiterinnen, als sogenannte Cloud-Worker, nicht nur an frei wählbaren Orten zu arbeiten, sondern auch zu frei wählbaren Zeiten (Apt et al. 2016, S. 18). Flexibilität prägt den mobilen Arbeitsplatz in einer zeitlichen und einer räumlichen Dimension. Man spricht somit auch von ,entgrenztem Arbeiten“ (BMAS 2015, S. 2; Francis \& Scheers 2013, S. 201f.). Viele schätzen es, gerade den Pendelaufwand $\mathrm{zu}$ reduzieren (BMAS 2015, S. 13). Sie sind durch diese Vorteile nicht nur zufriedener mit ihrem Job, sondern weisen auch eine höhere Verbundenheit zum Unternehmen auf. (White 2012, S. 207; BMAS 2015, S. 15). Dies gilt vor allem für die sogenannte Generation $\mathrm{Y}$, die zwischen 
1980 und 1995 geboren, nun auf den Arbeitsmarkt strömt (Bund 2014, S. 7). Auf der Basis von Generationenmodellen, wie sie Kupperschmidt (2000) vorschlägt, zeichnet sich diese u. a. durch die selbstverständliche Nutzung von Informationstechnologie aus. Sie möchten zu flexiblen Zeiten und selbstbestimmt arbeiten (Bund 2014, S. 56f.; White 2012, S. 205).

Andererseits bedeutet die Entgrenzung der Arbeit auch eine nicht immer erwünschte Vermischung von Arbeits- und Privatleben, was das Stressempfinden bei Angestellten erhöhen kann (Francis \& Scheers 2013, S. 201f.). Des Weiteren fürchten manche, dass die Zusammenarbeit mit Kollegen auf Dauer leidet, wenn der direkte Kontakt abnimmt (BMAS 2015, S. 13).

Einen elementaren Bestandteil des digitalen Arbeitsplatzes bilden Social-Intranet-Lösungen. Sie führen als moderne Intranet-Plattformen durch eine Erweiterung mit Komponenten aus sozialen Medien zu völlig neuen Möglichkeiten für die Zusammenarbeit und den Austausch: Angestellte können eigene Profile anlegen und mit personenbezogenen Informationen über sich füllen (Byström 2011). Über integrierte Anwendungen sind sie in der Lage diese Informationen nicht nur zu teilen, sondern auch miteinander zu kommunizieren, gemeinsam an Projekten zu arbeiten, Gruppen für Problemlösungen zu gründen, aber auch ihr Wissen und ihre Dokumente abzulegen und anderen zur Verfügung zu stellen (Ahmad \& Widén 2015). Widén diskutiert Ansätze, die das Teilen von Information modellieren können: Information Behavior, Social Capital, und Information Culture (Widén 2017).

Aber auch für das Wissensmanagement ergeben sich aus Social Tools Vorteile: Mitarbeiterinnen, die sich zuvor nicht miteinander austauschen konnten, obwohl sie Expertinnen auf dem gleichen Gebiet sind, ermöglichen Social Tools nun einen Transfer ihres Wissens. Der Unterschied der Social Tools im Gegensatz zu früheren Ansätzen des Wissensmanagements besteht in der persönlichen Weitergabe von Wissen. Früher wurde Wissen durch Dokumentation und Speicherung in Form von Daten mit dem Ziel konserviert, Unternehmenswissen zu sammeln, z.B. für den Fall, dass der Konzern Beschäftigte verliert. Das Problem dabei ist, dass implizites Wissen darüber verloren geht, wie das Wissen konkret auf die Arbeit angewandt werden kann (Ehrlich \& Lin 2011, S. 288f.). Social Software kann dieses Problem lösen, indem durch den persönlichen Kontakt komplexes Wissen weitergegeben werden kann. Es entsteht eine gesteigerte Innovationskraft durch Nutzung der „kollektiven Intelligenz“ der Masse (Buhse 2011, S. 89f.; Francis \& Scheers 2013, S. 201). In der Forschung wird zunehmend die Suche nach Expertinnen bearbeitet (Moreira et al. 2015).
Durch die „Vernetzungskraft sozialer Medien“ können Beschäftigte zudem ihr Potential und ihre Kompetenzen transparenter und kollaborativer präsentieren und nutzen, ohne dabei von einer Führungskraft „ausgebremst“ zu werden. Dies kann sich zusätzlich positiv auf die Innovationsfähigkeit und Kreativität des Unternehmens auswirken (Boes et al. 2015, S. 63; Buhse 2011, S. 89), deren Bedeutung für die Wettbewerbsfähigkeit in den letzten Jahren stark zugenommen hat (Schweer \& Seidemann 2015, S. 131). Durch die vereinfachte Informationsversorgung und das entgrenzte Arbeiten wird die Transparenz erhöht und zwar global über alle Wertschöpfungsprozesse und Hierarchieebenen hinweg (Francis \& Scheers 2013, S. 200). Informationen, die einst nur dem Management zur Verfügung standen, können nun von allen Mitarbeitenden genutzt werden.

Dies ermöglicht viel eigenständigere Entscheidungsprozesse, ohne dass auf Informationen aus anderen Hierarchieebenen gewartet wird. Dadurch sind auch Großunternehmen im Stande sich in flacheren Hierarchien zu organisieren und Vorteile wie eine gesteigerte Motivation und Kreativität der Angestellten sowie eine bessere Flexibilität der gesamten Organisation zu nutzen (Bollier 2011 S. 7). Insgesamt kann der vereinfachte Zugriff auf Informationen $\mathrm{zu}$ einer transparenteren und offeneren Unternehmenskultur führen, in der Beschäftigte ihr Wissen und ihre Fähigkeiten kollaborativ und kollektiv einsetzen, um ihre Aufgaben eigenständiger zu erledigen (Byström 2011).

Durch die neuen Technologien der Digitalisierung verbessert sich der Zugriff auf Information im Informationsund Wissensmanagement. In diesem Kontext wird oft vom sogenannten Informationsparadoxon gesprochen. Auf der einen Seite erwachsen immer bessere Zugriffsmöglichkeiten auf immer mehr strukturierte (Faktendaten) und unstrukturierte Informationen (Texte, Bilder oder Videos). Damit steigt der Umfang des Wissens, das zur Verfügung steht (Zheng et al. 2011, S. 1902). Auf der anderen Seite wird es, trotz dieses Angebotes, immer schwerer, die richtigen, den Bedürfnissen entsprechenden Informationen zu finden, den Zugriff zu teilen und darüber hinaus die Qualität der Informationen zu erhalten. Es muss immer mehr Zeit investiert werden, um das Informationsbedürfnis zu befriedigen und damit kann die Produktivität der Arbeit sinken (Zheng et al. 2011, S. 1901).

Für dieses Problem bietet die Digitalisierung technologische Lösungen. Die Stichworte lauten Big Data und Enterprise-Search. Die Enterprise-Suche ermöglicht eine Suche über alle Datenbestände im Unternehmen (Kruschwitz \& Hull 2017). Im Gegensatz dazu waren früher oft nur Suchabfragen in fragmentierten Datenbeständen möglich, die sich auf einzelne Datenbanken bezogen. Der begrenzte 
Informationszugriff hat damit einhergehend die Entscheidungsfähigkeit reduziert. Dank der zunehmenden Vernetzung und Transparenz der IuK-Technologien erhalten Angestellte über die Enterprise-Search, die oft in IntranetLösungen integriert ist, umfassende Suchergebnisse. Dies gilt insbesondere, da mittlerweile auch unstrukturierte Daten maschinenlesbar und -auswertbar werden (Benjamins 2014; Ehrlich \& Lin 2011, S. 292f.).

Durch stetig bessere Algorithmen werden die Suchergebnisse zudem fortlaufend optimiert. So haben sich z.B. Zheng et al. mit der Problematik auseinandergesetzt, dass es im Ranking der obersten Suchergebnisse oft zu Redundanzen kommt und dadurch die Varianz einer Suchanfrage nicht abgedeckt wird. Konsequenterweise haben Zheng et al. in ihrer Arbeit untersucht, wie die Diversität der Suchergebnisse verbessert werden kann (Zheng et al. 2011). Ähnliche Fortschritte gelten auch für Big Data. Wo früher nur Ausschnitte der verteilten Unternehmensdaten zur Verfügung standen, ermöglichen die IuK-Technologien, wie z.B. das maschinelle Lernen und das Cloud-Computing, die Analyse großer Datenmengen. Das Resultat sind präzisere Ergebnisse, wie z. B. Prognosen, die für Entscheidungsfindung genutzt werden können (Benjamins 2014).

Dieser Beitrag möchte einen umfassenden Blick auf Veränderungen am Arbeitsplatz geben, ohne dabei auf bestimmte Branchen oder Arbeitsbereiche zu fokussieren. Wie sehen die Veränderungen vom klassischen hin zum digitalen, wissensorientierten Arbeitsplatz aus? Einen Einblick aus Sicht der Führungs- und Entscheidungsebene werden dazu die folgenden Abschnitte liefern. Sie beleuchten Möglichkeiten und Chancen der Digitalisierung. Zudem zeigen sie, weshalb die Digitalisierung kein kurzzeitiges Trendthema ist, dem Unternehmen sich verwehren können, sondern eine Grundvoraussetzung für zukunftsorientiertes Handeln.

\section{Methodisches Vorgehen: Fokusgruppe}

In der vorliegenden Studie sollte die Perspektive der Unternehmen auf die Digitalisierung von Arbeitsplätzen ohne Vorannahmen erhoben werden, so dass eine qualitative und freie Form des Forschungsdesigns gewählt wurde. Am besten geeignet für derartige Fragestellungen ist eine Fokusgruppe. Eine Fokusgruppe ist mehr als nur ein Gruppen-Interview, da nicht nur Antworten auf die Fragen des Interviewers gegeben werden. Vielmehr stellt sich eine Mischung aus Meeting und Gespräch ein, so dass eine Diskussion zwischen den Teilnehmenden zu Stande kommt
(Jordan \& Wildemuth 2009). Die Stärke von Fokusgruppen liegt in der gegenseitigen Beeinflussung der Meinungsfindung von Teilnehmenden durch die Gruppenprozesse. Sie profitieren von der wechselseitigen Kommunikation und dem Austausch von Gedanken, die ihr eigenes Wissen um das der Anderen erweitern. Als Gruppenleistung können bessere oder neue Ideen oder Konzepte entstehen.

Als Homogenitätskriterium für diese Studie wurde beschlossen, die potentiellen Teilnehmenden auf die Führungs- und Entscheidungsebene einzugrenzen, die an der Gestaltung von IT, Informationsinfrastrukturen, Informationszugängen und dem digitalen Arbeitsplatz im allgemeinen verantwortlich oder zumindest daran beteiligt sind. Grund dafür war, dass diese Verantwortung für Digitalisierungsprozesse tragen und sich daher bewusster mit Hürden und Herausforderungen in der Umsetzung beschäftigen als normale Angestellte. Zudem wurden Beschäftige aus Großkonzernen bevorzugt. Insgesamt nahmen sechs Personen an der Fokusgruppe teil.

Die initialen Themen für die Fokusgruppe wurden in einschlägigen Blogs (z.B. digitalworkplacegroup.com, blog.enterprise-digital.net, socialmedia4dialog.wordpress .com) gesammelt und nicht in der Forschungsliteratur, um nahe an der Terminologie der Unternehmen zu bleiben. Das Vorgehen ähnelte der Blog-Analyse von Antes (2015). Anschließend erfolgten drei Experteninterviews, um zu überprüfen, inwieweit die identifizierten Aspekte relevant sind und die Thematik abdecken. Diese wurden als leitfadengestützte telefonische Einzelinterviews (Misosch 2014, S. 124 f.) durchgeführt.

In einer Fokusgruppe dient eine Eröffnungsfrage dem Auflockern der Stimmung und der Steigerung der Teilnahme. Hier geht es um eine möglichst frühe aktive Beteiligung aller Teilnehmenden (Krueger \& Casey 2000, S. 44). Als „Eisbrecher“ für die Eröffnung wurde die folgende Frage gewählt: „Wer von Ihnen hat heute schon sein Smartphone für berufliche Zwecke genutzt und für was?“ Im Folgenden wurden zwei Kernblöcke mit dazu gehörigen Schlüsselfragen diskutiert.

Block 1: Wie verändern sich Ihrer Erfahrung nach durch die zunehmende Digitalisierung der Austausch und die Nutzung von Information durch die Mitarbeitenden am Arbeitsplatz?

- Arbeiten von überall und zu jeder Zeit

- Unterschiede in der digitalen Kompetenz und ihre Auswirkungen auf Wissensaustausch

- Herausforderungen für Beschäftigte bei der Suche und Nutzung von Information

- Herausforderungen für Angestellte bei der Verwaltung ihrer Informationen (Persönliches Informationsmanagement) 
- Möglichkeiten zur Nutzung von Big-Data für einzelne Mitarbeiter und Mitarbeiterinnen

- Möglichkeiten der Automatisierung für den Umgang mit der Informationsflut

Block 2: Wie verändern sich Ihrer Erfahrung nach durch die zunehmende Digitalisierung die Zusammenarbeit und die Organisationsstrukturen in Ihrem Unternehmen?

- Zu welchem Grad ist Social Software integriert?

- Welche Herausforderungen entstehen durch die $\mathrm{Zu}-$ sammenarbeit in wechselnden Projektzusammenhängen?

- Entsteht Konfliktpotential zwischen Social Software und Führungsstilen?

- Zusammenarbeit mit externen Stakeholdern

- Erfolgsbewertung von Informationssystemen

Um die Fokusgruppe später analysieren zu können, wurden mit Einverständnis der Teilnehmenden Audioaufnahmen erstellt und zusätzlich Protokolle der gesamten Sitzung erstellt. Als Protokollart bot sich u.a. das Verlaufsprotokoll an, da hierbei die Äußerungen chronologisch dem jeweiligen Sprecher zugeordnet sind und damit eine Nachvollziehbarkeit des Diskussionsverlaufs mit den jeweiligen Standpunkten gewährleistet ist (Langer 2009, S. 14). Details zur Transkription finden sich in Schwab (2017).

Sowohl die Analyse der Blogs, der Experteninterviews als auch der Transkripte der Fokus-Gruppe erfolgten nach Methoden der qualitativen, deduktiven Inhaltsanalyse (Mayring 2010, S. 65). Die gefundenen Kategorien sind in den folgenden Tabellen mit „K“ gekennzeichnet.

\section{Ergebnisse: Problemfelder der Digitalisierung aus Sicht der Unternehmen}

Das Ergebnisprotokoll zeigt, dass für die Teilnehmenden die folgenden Themen im Fokus standen:

- Unternehmenskultur und Transparenz (nicht jeder Mitarbeiter wird mitgenommen)

- Mängel in der Medien- und Informationskompetenz

- Generationsproblem (begrenzte Bereitschaft älterer Beschäftigter für Veränderungen, Silo-Denken in Bezug auf Teilen von Wissen und Information)

- Uneinheitliche Strukturen im Informationsmanagement (zu viele Altlasten und Datenmüll in Systemen)

- Veraltete Strukturen und Standards, während sich die Unternehmensführung von Angestellten konträr dazu ein modernes Startup-Denken wünscht
- Negativer Umgang mit Feedback und Fehlerkultur

- Soft-Skills der Führungskräfte

- Work Roles sind zu starr festgeschrieben / fehlendes Personal.

Die wichtigsten Herausforderungen aus Sicht der Teilnehmenden waren die folgenden:

- Technik an Nutzeranforderungen anpassen: „Kulturwandel durch gute Technik"!

- Unternehmenskultur durch Vorleben und richtige Strukturen ändern!

- Medien- und Informationskompetenz fördern (verpflichtend für alle)!

- Veränderungen konsequent fördern und aktiv umsetzen!

Bei den Chancen der Digitalisierung wurden zuallererst die Möglichkeiten für Kommunikation und Austausch genannt. Dazu zählten das entgrenzte Arbeiten über Länderund Zeitgrenzen hinweg. Dies führe zu einer besseren Verfügbarkeit von Wissen im ganzen Unternehemen, unabhängig von Hierarchien und zu mehr Transparenz und Synergien bei der Zusammenarbeit. Weitere konkrete Ausprägungen der Chancen in diesem Bereich, die genannt wurden, lassen sich der Tabelle 1 entnehmen, welche die Nennungen auch quantitativ auswertet. Die Häufigkeiten beziehen sich auf die Frequenz der Nennung des Themas im Lauf der Diskussion der Fokusgruppe.

Die Studie zeigt, dass sehr verschiedenartige digitale Arbeitsplätze anzutreffen sind und diese jeweils erfordern, dass viele verschiedene Systeme gemanagt werden. Dieses Überangebot an Systemen verunsichert manche und lässt sie im Unklaren darüber, welches System für die Bewältigung einer Aufgabe am besten geeignet ist. Darüber hinaus sind gegenwärtig, wenn überhaupt, nur Teile der vielen Systeme miteinander verbunden. Diese mangelhafte Systemintegration mindert die Bedienbarkeit.

Das Trendthema „Einsatz mobiler Geräte“ stand bei den Unternehmen auf der Agenda, ist also in Planung oder wird umgesetzt, um ortsungebundenes Arbeiten zu ermöglichen. Denn gegenwärtig sei bei einem Großteil der Teilnehmenden der digitale Arbeitsplatz nach wie vor sehr desktop-orientiert. Es werde geprüft, ob das mobile Arbeiten mit Bring-your-own-Device kombiniert werden kann, weil dies zunehmend gefordert wird. Die Realisierung des mobilen Arbeitens laufe aber noch schleppend: Es konnte nur ein Teilnehmer berichten, dass mobiles Arbeiten tatsächlich schon produktiv umgesetzt wurde. Als Gründe wurden genannt, dass in großen Unternehmen mit vielen Bereichen erst der Nutzen geprüft werden müsse. Nicht alle Bereiche seien für mobiles Arbeiten 
Tabelle 1: Wichtige genannte Themen und die Häufigkeit der Nennung.

\begin{tabular}{|c|c|c|}
\hline Kategorie & Unterkategorie (Anzahl der Nennungen) & $\begin{array}{l}\text { Anzahl der } \\
\text { Nennungen }\end{array}$ \\
\hline $\begin{array}{l}\text { K15 } \\
\text { Kollaboration, } \\
\text { Austausch, } \\
\text { Kommunikation } \\
\text { über Tools }\end{array}$ & $\begin{array}{l}\text { a) Intern } \\
\text { - Austausch über Länder- und Zeitzonengrenzen hinweg (2) } \\
\text { - Transparenz von Informationen über Hierarchieebenen und Abteilungen hinweg: } \\
\text { weniger Hoheitswissen (5) } \\
\text { - Beschäftigte können entscheiden, wann sie welche Information konsumieren (1) } \\
\text { - Einfacheres Teilen von Information (1) } \\
\text { - Transparenz \& Synergien: Zusammenarbeit und Austausch über Abteilungen und } \\
\text { Hierarchien hinweg (6) } \\
\text { - Mehr Innovationen (1) } \\
\text { - Blog als Ablage / Quelle für Wissen (1) } \\
\text { b) Extern } \\
\text { - Kunden gewinnen (2) } \\
\text { - Suchmaschinenoptimierung (1) } \\
\text { - Transparenz (1) }\end{array}$ & $\begin{array}{l}\text { Gesamt: } 21 \\
\text { a) } 17 \\
\text { b) } 4\end{array}$ \\
\hline $\begin{array}{l}\text { K16 } \\
\text { Wissens- } \\
\text { management }\end{array}$ & $\begin{array}{l}\text { - Es steht mehr Wissen zur Verfügung (1) } \\
\text { - Extern frei verfügbares Wissen: positiver Zwang, sich fortbilden zu müssen (1) } \\
\text { - Kommunikationsverläufe haben Mehrwert als Informations- und Wissensquelle (1) }\end{array}$ & Gesamt: 3 \\
\hline $\begin{array}{l}\text { K17 } \\
\text { Generationen }\end{array}$ & Digitale Kompetenzen junger Mitarbeitender ermöglichen den digitalen Wandel (2) & Gesamt: 2 \\
\hline $\begin{array}{l}\text { K18 } \\
\text { Technologie }\end{array}$ & $\begin{array}{l}\text { Kulturwandel durch gute Technologie (3) } \\
\text { - Mehr Austausch / Transparenz durch gute Technologie (1) } \\
\text { - Nicht die technischen Einschränkungen der Systeme, sondern ihre Integration in die Prozesse } \\
\text { führt zu Problemen (5) }\end{array}$ & Gesamt: 9 \\
\hline $\begin{array}{l}\text { K19 } \\
\text { System- } \\
\text { Evaluation }\end{array}$ & $\begin{array}{l}\text { Evaluation spart auf lange Sicht Kosten (2) } \\
\quad \text { - Schnellere Einführung von alternativen Systemen / Systemanpassungen (3) }\end{array}$ & Gesamt: 5 \\
\hline
\end{tabular}

geeignet: z.B. könnten Beschäftigte im technischen Außendienst kein Homeoffice nutzen. Zudem würde der Fortschritt im Bereich „Mobile“ durch das Thema Datenschutz erschwert. So bestehe zwar der Bedarf für Kommunikation über Apps auf mobilen Endgeräten. Dies sei erkennbar z.B. an der hohen Nutzungsrate von WhatsApp für berufliche Zwecke. Doch ist dies schon allein aus Datenschutzgründen umstritten.

Der erste Hauptteil der Fokusgruppe beschäftigte sich neben den oben angesprochenen Chancen auch mit den Hürden und Herausforderungen für das Informationsmanagement, mit denen die einzelnen Angestellten bei der Nutzung und dem Austausch von Information am digitalen Arbeitsplatz konfrontiert werden. Tabelle 2 zeigt die identifizierten Hürden, gruppiert als Kategorien in absteigender Reihenfolge nach Anzahl der Nennungen.
Tabelle 2: Wichtigste genannte Hürden für individuelle Arbeitsplätze und die Häufigkeit der Nennung.

\begin{tabular}{llr}
\hline Rang & Kategorie & $\begin{array}{r}\text { Anzahl der } \\
\text { Nennungen }\end{array}$ \\
\hline 1 & K8 Systeme & 31 \\
\hline 2 & $\begin{array}{l}\text { K5 Strukturen im Informations- und } \\
\text { Wissensmanagement }\end{array}$ & 25 \\
\hline 3 & K7 Generationsproblem: Demografische & 20 \\
\hline 4 & Entwicklung & 18 \\
\hline 5 & K10 Führung & 18 \\
\hline 6 & K9 Unternehmenskultur & 11 \\
\hline 7 & Mitarbeitenden & 6 \\
\hline 8 & K14 Externe oder indirekte Faktoren & 2 \\
\hline
\end{tabular}

Die wichtigsten fünf Hürden werden im Folgenden noch detailliert diskutiert. 
Nach der Auszählung der Daten hat sich überraschenderweise ergeben, dass die größte Hürde, auf die Beschäftigte beim persönlichen Informationsmanagement stoßen, System-Mängel darstellen. Überraschend ist der Befund deswegen, weil mehrfach geäußert wurde, dass die Herausforderungen und Barrieren nicht technologischer Natur seien. Bei genauer Betrachtung der Gründe, weshalb Systeme die größte Hürde darstellen, bestätige sich, dass es in den meisten Fällen nicht an der technischen Machbarkeit an sich scheitere. Vielmehr lägen die Ursachen im Umgang mit der Technik oder an unzureichenden Ausschöpfung der Möglichkeiten. Beispielsweise seien mangelhaftes Design und die oft als schlecht bewertete Usability der Systeme keine technologischen bzw. zumindest keine funktionalen Aspekte. Als wichtige Ursache wurde häufig genannt, dass keine Evaluation vor einer Systemeinführung stattfindet und zwar meist aus Zeitgründen. Zudem stehe kein adäquates Personal zur Verfügung, um die Mängel an den Systemen zu beheben. Da diese Aufgaben als zu anspruchsvoll wahrgenommen werden und Rollen der Beschäftigten starr festgeschrieben seien, ließe sich intern kaum jemand für solche Aufgaben finden. Zudem schränke eine schlechte Integration von verschiedenen Systemen die Funktionalität ein.

Als zweitgrößte Hürde werden mangelhafte Strukturen im Informations- und Wissensmanagement genannt. Es fehlen im Umgang mit Informationen Vorgaben, wo und wie diese richtig abgelegt und geteilt werden können. Zudem mangele es an einer Übersicht darüber, welche Informationen überhaupt vorhanden sind und wie sich diese Informationen im Konzern bewegen (z. B. wer hat Zugriffsrechte?). Insbesondere verschärfe sich das Problem bei der Projektarbeit, wenn aufgrund fehlender Synergien in jedem Projekt intern eigene Strukturen geschaffen werden. Das Vorhandensein von Strukturen allein reiche nicht aus, wenn Kompetenzen im Umgang mit diesen Strukturen und Informationen fehlen. Konkret genannt wurden Schwierigkeiten beim Klassifizieren, Indexieren, der Relevanzbewertung und im Umgang mit den zur Verfügung stehenden Tools.

Mit 20 Nennungen wurden demografische Herausforderungen am dritthäufigsten thematisiert. Ob und inwieweit das Alter eine Rolle spielt, blieb dabei umstritten. Diese Kategorie wird teilweise in Verbindung mit der Kategorie Informations- und Medienkompetenz der Beschäftigten genannt. Wenn die Teilnehmenden eine Abhängigkeit vom Alter der Mitarbeitenden gesehen haben, dann aus folgenden Gründen:

- Fehlerkultur: Ältere Angestellte befürchten, beim Umgang mit Systemen Fehler zu machen. Daher fehle ihnen die Neugier, um neue Systeme zu erkunden und deren Bedienung selbstständig zu erlernen.

- Keine ausreichende Informations- und Medienkompetenz älterer Beschäftigte.

- Silodenken und mangelnde Transparenz: Ältere Generationen zeigen eine geringe Bereitschaft, ihr Wissen preiszugeben und mit Kollegen $\mathrm{zu}$ teilen, aus Angst, unnütz $\mathrm{zu}$ werden und damit einhergehend ihren Job oder ihre Position zu verlieren.

- Keine Bereitschaft für Digitalisierung: Mit zunehmendem Alter sinke die Bereitschaft Neues zu lernen.

Als Hürde an vierter Stelle wurde die Führung in der Diskussion genannt. Am häufigsten wurde kritisiert, dass von den Führungsebenen nicht genug dafür getan wird, um alle Mitarbeitende für den Digitalisierungsprozess $\mathrm{zu}$ gewinnen. Statt Hilfe anzubieten, mit gutem Beispiel voranzugehen oder fehlende Informationskompetenzen zu vermitteln, akzeptierten viele Führungskräfte einfach das „Nichtkönnen“ oder „Nichtwollen“ ihrer Beschäftigten. Diese würden nicht ausreichend zu Lernprozessen angeregt und darin gefördert - sei es durch eigenständiges Lernen, indem sie sich selbst mit der Digitalisierung auseinandersetzen - oder in Bezug auf ihre Pflicht, lebenslang zu lernen, um ihren Job auch in Zukunft fachmännisch ausüben zu können.

Probleme in der Unternehmenskultur (Platz 5) entstehen laut einigen Aussagen vorrangig in alten, historisch gewachsenen Unternehmen. Durch Zukäufe bildeten sich an vielen Stellen Subkulturen im Unternehmen, die Synergien zwischen den Bereichen vereiteln. Zudem seien die Kulturen insgesamt oft von veralteten Strukturen geprägt, die die Unternehmen gerne aufbrechen möchten. Zum einen vernachlässige ein Teil der Angestellten die Auseinandersetzung mit dem Thema Digitalisierung, wodurch Nutzen und Chancen nicht erkannt werden. Zum anderen herrscht eine generelle Abschottung gegenüber digitalen Werkzeugen und Prozessen, also keinerlei Bereitschaft, Veränderungen und den Fortschritt zuzulassen. Besonders schwerwiegend sei das kulturelle Problem des Silodenkens. Zudem sei u.a. der Arbeitsbereich entscheidend: In einigen Abteilungen sei die Nutzung digitaler Werkzeuge üblicher als in anderen. Hier spiele auch eine wichtige Rolle, inwieweit die Nutzung durch Führungskräfte vorgelebt wird und ob der Arbeitsbereich international bzw. national organisiert ist. International arbeitende Teams seien wesentlich digitaler aufgestellt, um sich besser über Landes- und Zeitgrenzen hinaus austauschen $\mathrm{zu}$ können. Bei nationalen Teams bestehe hingegen oft die Hürde, dass die Beschäftigten den Mehrwert nicht sehen, da ja persönlicher Kontakt möglich ist. Darüber hinaus übe die 
vorherrschende Kultur im Unternehmen Einfluss darauf aus, wie Angestellte mit der Digitalisierung umgehen.

\section{Organisatorische Perspektive}

Im zweiten Hauptteil der Fokusgruppe wurden die Hürden und Herausforderungen in der Organisation und Zusammenarbeit im Unternehmen besprochen (s. Tab. 3).

Tabelle 3: Wichtigste genannte Herausforderungen in der Organisation.

\begin{tabular}{llr}
\hline Rang & Kategorie & $\begin{array}{r}\text { Anzahl der } \\
\text { Nennungen }\end{array}$ \\
\hline 1 & K10 Führung- \& Unternehmenskultur & 18 \\
\hline 2 & K9 Unternehmenskultur & 11 \\
\hline 3 & K14 Externe oder indirekte Faktoren & 10 \\
\hline 4 & K11 Strukturen und Arbeitsweisen & 6 \\
\hline 5 & K8 Systeme & 4 \\
\hline 6 & K12 Organisation - Personalabteilung (HR) & 3 \\
\hline 7 & K13 Strukturen für Kollaboration / Austausch & 2 \\
\hline
\end{tabular}

Als schwierigste Barriere nannten die Teilnehmenden erneut den Führungsstil der Vorgesetzten. Dazu gehört auch, dass Führungskräfte zu selten bei der Nutzung von neuen Systemen und Social Tools, wie z. B. Blogs, mit gutem Beispiel vorangehen. Dieser Mangel resultiere zum Teil aus fehlenden eigenen Kompetenzen der Führungskräfte im Umgang mit den Tools.

Zudem wurde geäußert, dass die Führung einen Teil ihrer Kontrollmacht über die Unternehmenskommunikation an die Belegschaft abgeben muss. Nur so können echte Dialoge auf Augenhöhe mit und unter den Beschäftigten entstehen und das Potenzial, das in Social Tools steckt, voll genutzt werden.

Neben dem Kernbereich Hürden und Herausforderungen in der Führung wurde das Thema „Unternehmenskultur" und dazugehörend vor allem die Fehlerkultur besprochen. Als negative Fehlerkultur seien alle Ausprägungen zu sehen, die dazu führen, dass sich Angestellte nicht an der öffentlichen Diskussion über Social Tools beteiligen, weil sie Angst haben, sich zu blamieren, oder sich vor Schuldzuweisungen fürchten. Darüber hinaus reduziere eine negative Fehlerkultur auch die Bereitschaft für System-Evaluationen innerhalb der Organisation, weil Verantwortliche nicht die Konsequenzen für Fehler und Misserfolge tragen möchten. Die Folge seien schlechte IT-Systeme, die ebenfalls die Digitalisierung am Arbeitsplatz erschweren.
Ferner wurde auf Hürden für Abläufe in der Organisation eingegangen: Es würden nicht ausreichend Rahmenbedingungen für Innovationen wie z. B. für das entgrenzte Arbeiten geschaffen. Stattdessen bliebe es oft bei alten und bewährten Strukturen und Arbeitsweisen, weil Risiken gescheut werden. Zudem gäbe es Barrieren in der interdisziplinären Zusammenarbeit, weil es u.a. an Personal mit den nötigen Kompetenzen fehle oder die Vernetzung aller Mitarbeiterkompetenzen im Unternehmen aufgrund fehlender Strukturen für die Zusammenarbeit schwierig sei.

Neben diesen internen Barrieren würden Organisationen auch mit äußeren Faktoren konfrontiert wie veränderten Kundenanforderungen bei der Nutzung von Systemen, auf die nur geringfügig Einfluss genommen werden kann. Hier müssten sich Unternehmen intern so aufstellen, dass sie auf diese Herausforderungen reagieren können.

\section{Diskussion und Vergleich mit der Literatur}

Die erzielten Ergebnisse lassen sich gut mit einer Studie von Katriina Byström aus dem Jahr 2011 vergleichen, die zu ähnlichen Ergebnissen kam. Dabei ging es um die Frage, welche Hürden Beschäftigte bei der Nutzung von neu implementierten Social Tools wahrnehmen (Byström 2011). Byström berichtet ebenfalls von der Hürde der Fehlerkultur oder davon, dass der Bedarf und Nutzen eines Social Tools nicht gesehen oder generell keine Bereitschaft besteht, neue Tools zu erlernen (Byström 2011, S. 2f.).

Ähnliche Erkenntnisse wie die aus der Fokusgruppe werden auch in anderen Publikationen angesprochen. Beispielsweise gibt es eine Analyse der Intranet-Einführung bei der Robert Bosch GmbH (Buhse 2014, S. 14f.). Auch hier zeigte sich der Führungsstil als Hürde bei der Umsetzung. Die Führungskräfte betrachteten das Projekt als reines ITProjekt, das technisch neue Funktionen zur Verfügung stellt. Die Einsicht, dass damit aber auch der Verlust von alten Mechanismen in Bezug auf Hierarchie und Kontrolle einhergeht, folgte erst langsam. Die Führungskräfte mussten erst das „Management by Internet“ lernen, d.h. ein neues Mind-Set aufbauen, das von Werten wie „Vernetzung, Offenheit, Partizipation und Agilität“ geprägt ist (Buhse 2014, S. 14). Auch hier zeigt sich, dass für den digitalen Wandel nicht technische, sondern kulturelle Veränderungen im Vordergrund stehen und zwar vorrangig im Führungsstil (Buhse 2014, S. 14f.):

- Angestellten mehr Spielraum für Selbstorganisation geben, Abbau von Regelungen und Bürokratie. 
- Mehr Experimentierfreude an den neuen Mechanismen und damit einhergehend auch eine Veränderung der Fehlerkultur schaffen. Es muss sich eine Bereitschaft entwickeln, auch Fehler in Kauf zu nehmen.

- Durch junge Beschäftigte (Digital Natives) kommt quasi automatisch Potenzial für den digitalen Wandel in das Unternehmen. Digital Natives bringen neue Arbeitsweisen und Ideen mit. Führungskräfte müssen lernen, dieses Potenzial zu nutzen und müssen diese mehr in Entscheidungsprozesse einbinden.

Diese Veränderungen müssen von Führungskräften geleitet werden, um eine unternehmensweite Umsetzung zu garantieren. Die Bedeutung des Themas in der Fokusgruppe zeigt sich auch in der Forschungsliteratur.

Durch die zunehmend allgegenwärtige Verfügbarkeit sind alle Hierarchieebenen besser mit Informationen versorgt und dazu zählt auch Information, die sonst nur für das Management bereitstand. Diese steigende informatorische Transparenz bedeutet, dass sich Wissen für Entscheidungsprozesse zunehmend dezentral und demokratischer auf viele verteilt, anstatt nur auf einige wenige Führungskräfte bzw. Wissensträger (Welpe et al. 2015, S. 78 f.). Dies kann einen entscheidenden Vorteil darstellen, da einige wenige Führungskräfte in immer stärker global agierenden Großkonzernen nicht mehr in der Lage sind, alle Entscheidungen allein zu steuern. Die Menge an Informationen übersteigt irgendwann die Kapazität eines einzelnen Menschen (Bollier 2011, S. 19).

Jedoch ist die Organisation der Zusammenarbeit innerhalb von flachen Hierarchien sehr schwierig. Es ist ein höheres Maß an Vernetzung nötig, da sich Arbeitsgruppen immer häufiger interdisziplinär und in wechselnden Konstellationen aufstellen (Schweer \& Seidemann 2015, S. 133). Dadurch befinden sich Führungsebenen im Umbruch. Ihre Aufgabenfelder verschieben sich. Diese Herausforderungen wurden auch in der Fokusgruppe diskutiert. Eine Herausforderung, die diesbezüglich häufig formuliert wurde, ist, dass Vorgesetzte die Digitalisierung „Vorleben“ müssen. Ebenso sollten sie die Nutzeranforderungen aufgreifen. Die primäre Rolle der Führungskräfte verschiebt sich von einer delegierenden autoritären Rolle hin zu der eines „Coaches und Mentors“ (Schweer \& Seidemann 2015, S. 134). Statt im klassischen Sinne zu führen, müssen sich Führungskräfte mehr um die Motivation und Förderung ihres Teams kümmern, um nicht nur die ganze Belegschaft im Digitalisierungsprozess mitzunehmen, sondern auch deren Kompetenzen stetig und langfristig auszubauen.

Durch diese veränderte Rolle werden sich auch die Informationsbedürfnisse der Führungsebenen verändern.
Zudem müssen Führungskräfte sich damit auseinandersetzen, neue Rahmenbedingungen zu schaffen und deren Einhaltung zu kontrollieren. Ein Beispiel ist die Erreichbarkeit der Beschäftigten beim entgrenzten Arbeiten. Wenn es keine Arbeitszeiten mehr gibt, haben diese dann auch ein Recht auf Nicht-Erreichbarkeit und wann gilt es? Oder wo endet dann die Verantwortung des Arbeitgebers in Bezug auf Sicherheit und Gesundheit am Arbeitsplatz (Nahles 2015, S. $27 \mathrm{f}$.)?

Damit werden Führungskräfte zunehmend eine zentrale Vermittlerfunktion zwischen Angestellten und der technologischen Digitalisierung am Arbeitsplatz einnehmen. Sie müssen viele neue Fragen bezüglich der Arbeitsorganisation klären, denn auch für das Einhalten von übergeordneten Zielen, die den Erfolg des Unternehmens wahren sollen, werden zu Zeiten der Digitalisierung Führungskräfte gebraucht. Zu den übergeordneten Aufgaben werden weiterhin das Erstellen von Gesamtstrategien und Unternehmenszielen zählen (Schweer \& Seidemann 2015, S. 134). Gleichzeitig werden Beschäftigte immer mehr $\mathrm{Zu}$ griff auf die Informations- und Wissensflüsse haben und das Informations- und Wissensmanagement maßgeblich beeinflussen.

\section{Fazit und Ausblick}

Die Hürden und Herausforderungen, mit denen alle bei der Nutzung und dem Austausch von Information am digitalen Arbeitsplatz konfrontiert werden, sind in erster Linie Probleme mit Systemen, weil Design, Usability und Funktionalitäten mangelhaft sind. Dabei sind diese Probleme nicht technischer Art, sondern entstehen beim Umgang mit den Systemen. Darüber hinaus haben alle im persönlichen Informationsmanagement mit fehlenden oder mangelhaften Strukturen beim Umgang mit Information zu kämpfen: Es fehlt an Vorgaben, welche Informationen vorhanden sind, wo nach ihnen gesucht werden kann, wie und wo sie abzulegen sind oder wie man die Informationen mit anderen teilen kann. Zum Teil liegt dies aber auch an unzureichenden Kompetenzen der Beschäftigten, die den richtigen Umgang mit Systemen und/oder Informationen nicht beherrschen (z. B. Vorgehensweise der korrekten Indexierung).

Hier zeigen sich also klassische Themen der Informationswissenschaft. Suche und Informationsmanagement stehen dabei ganz oben, aber auch schlechte Usability tritt häufig auf. Daneben folgen Probleme des Informationsmanagements. Verschiedene Systeme sind oft schlecht integriert, weil zu wenig Zusammenarbeit und Absprache bei deren Konzeption und Einführung erfolgen. 
Nicht klar zu beantworten ist dabei die Frage, inwieweit das Alter der Beschäftigten Einfluss auf deren Informations- und Medienkompetenz und auch auf das Voranschreiten der Digitalisierung im Allgemeinen nimmt. Es wurde von demografischen Problemen berichtet, aber zeitgleich gab es auch Hinweise auf andere altersunabhängige Einflussfaktoren, so dass eine eindeutige funktionale $\mathrm{Zu}$ ordnung schwierig wurde. Als unabhängig vom Alter wurden z.B. die vorherrschende Kultur in den verschiedenen Arbeitsbereichen und die Förderung durch Vorgesetzte genannt.

Führung und Unternehmenskultur wurden auch fernab vom Generationsproblem als für sich stehende Hürden beschrieben, die das Informationsmanagement am digitalen Arbeitsplatz erschweren: z.B. werden nicht alle Beschäftigten genügend von den Führungsebenen gefördert, um den Anschluss in der Digitalisierung nicht zu verlieren.

Diese Forschung wird international fortgesetzt. Vergleichbare Fokusgruppen wurden in mehreren Ländern durchgeführt, um zu erkennen, welche Themen in welcher Kultur besonders von Bedeutung sind. Die vergleichende Auswertung steht noch aus.

\section{Literatur}

Ahmad, Farhan; Widén, Gunilla (2015): Language clustering and knowledge sharing in multilingual organizations: A social perspective on language. In: Journal of Information Science, 41(4), S. 430-443.

Antes, Kea (2015): Eine qualitative Inhaltsanalyse anhand ausgewählter Foodblogs. Hamburg: Hochschule für Angewandte Wissenschaften. http://edoc.sub.uni-hamburg.de/haw/voll texte/2015/3179/ [5.3.2018].

Apt, Wenke; Bovenschulte, Marc; Hartmann, Ernst A.; Wischmann, Steffen et al. (2016): Foresight-Studie Digitale Arbeitswelt. Bundesministerium für Arbeit und Soziales (BMAS) 2016. http:// www.bmas.de/DE/Service/Medien/Publikationen/Forschungs berichte/Forschungsberichte-Arbeitsmarkt/fb-463-digitale-ar beitswelten.html?nn=67546 [5.3.2018].

Benjamins, Richard (2014): Big Data. From Hype to Reality? In: Proceedings 4th International Conference on Web Intelligence, Mining and Semantics, Artikel Nr. 2, WIMS 2014. https://doi.org/ 10.1145/2611040.2611042

BMAS (2015): Bericht zum Monitor. Mobiles und entgrenztes Arbeiten. Forschungskooperation des Bundesministeriums für Arbeit und Soziales (BMAS) 2015. http://www.bmas.de/DE/Service/ Medien/Publikationen/Forschungsberichte/Forschungsbericht e-Arbeitsmarkt/fb-460-mobiles-und-entgrenztes-arbeiten.html [5.3.2018].

Boes, Andreas et al. (2015): Zwischen Empowerment und digitalem Fließband. Das Unternehmen der Zukunft in der digitalen Gesellschaft. In: Sattelberger, Thomas et al. (Hrsg.): Das demokratische Unternehmen. Freiburg u. a.: Haufe Gruppe, S. 57-73.
Bollier, David (2011): The Future of the Work. What it means for individuals, Businesses, Markets and Governments. Washington, D. C.: Communications and Society Programm 2011

Buhse, Willms (2014): Digital Leadership bei der Robert Bosch GmbH. In: Wissensmanagement. Das Magazin für Führungskräfte 6 (2014), S. 14-15.

Buhse, Willms (2011): Enterprise 2.0 im Management. Frischzellenkur für Unternehmen. In: Wolf, Frank (Hrsg.): Social Intranet. Kommunikation fördern, Wissen teilen, Effizient zusammenarbeiten. München: Carl Hanser Verlag 2011, S. 87-101.

Bund, Kerstin (2014): Glück schlägt Geld. Generation Y: Was wir wirklich wollen. Hamburg: Murmann.

Byström, Katriina (2011): Social media discomfort. The clash between the old and the new at work. In: Proceedings of the American Society for Information Science and Technology 1 (2011)

Byström, Katriina (2016): Information Challenges, Challenging Information in Digital Workplaces. Workshop report from ENWI Symposium 2015, September 29-30, Gothenburg, Sweden. Journal of Library and Information Sciences.

Byström, Katriina; Ruthven, lan; Heinström, Jannica (2016): Work and work information: Which workplace models still work in modern digital workplaces? In: Conceptions of Library and Information Science (CoLIS) June 26-29: Uppsala, Sweden. http://www.infor mationr.net/ir/22-1/colis/colis1651.html [5.3.2018].

Dirks, Thorsten (2016): Digitalisierung der Wirtschaft. Bitkom Research 2016 https://www.bitkom.org/Presse/Anhaenge-anPIs/2016/Maerz/Digitalisierung-Gesamtwirtschaft.pdf [5.3.2018].

Ehrlich, Stefan; Lin, Dada (2011): Das Social Intranet und seine Rolle für das Wissensmanagement. In: Wolf, Frank (Hrsg.): Social Intranet. Kommunikation fördern, Wissen teilen, Effizient zusammenarbeiten. München: Carl Hanser Verlag 2011, S. 287-301.

Francis, James; Scheers, Caroline (2013): The future workplace of young Europeans. In: European View 12 (2013). Center for European Studies. S. 199-204. http://link.springer.com/article/ 10.1007 \%2Fs12290-013-0286-y [5.3.2018].

Hermann, Mario; Pentek, Tobias; Otto, Boris (2015): Design Principles for Industry 4.0 Scenarios. A Literature Review. Technische Universität Dortmund Fakultät Maschinenbau/ Audi Stiftungslehrstuhl Supply Net Order Management 2015. http://www.thiagobranquinho.com/wp-content/uploads/ 2016/11/Design-Principles-for-Industrie-4_0-Scenarios.pdf [5.3.2018].

Jordan, Mary Wilkins; Wildemuth, Barbara (2009): Focus groups. In: Wildemuth, Barbara (ed.): Applications of social research methods to questions in information and library science. ABC-CLIO, Incorporated

Krueger, Richard A.; Casey, Mary Anne (2000): Focus groups. A practical guide for applied research. Sage Publication.

Kruschwitz, Udo; Hull, Charlie (2017): Searching the Enterprise. In: Foundations and Trends in Information Retrieval 11.1 pp.1-142.

Kupperschmidt, Betty (2000): Multigenerational Employees: Strategies for Effective Management. In. Health Care Manager vol. 19. S. $65-76$

Langer, Nicole (2009): Protokolle Mitschrift und Ausarbeitung optimieren. München: Compact 2009

Mayring, Philipp (2010): Qualitative Inhaltsanalyse. Grundlagen und Techniken. Weinheim: Beltz. 
Misosch, Sabrina (2014): Qualitative Interviews. Berlin: De Gruyter Oldenbourg 2014.

Moreira, Catarina; Calado, Pável; Martins, Bruno (2015): Learning to rank academic experts in the DBLP dataset. In: Expert Systems vol. 32.4: S. 477-493.

Nahles, Andrea (2015): Demokratie und Selbstbestimmung in der digitalen Arbeitswelt. Sozialstaatliche Rahmenbedingungen und politische Perspektiven. In: Sattelberger, Thomas et al. (Hrsg.): Das demokratische Unternehmen. Freiburg u. a.: Haufe Gruppe, S. 23-28.

Schwab, Ella (2017): Austausch und Nutzung von Information am digitalen Arbeitsplatz. Eine Analyse von Chancen, Herausforderungen und Barrieren. Masterarbeit Internationales Informationsmanagement, Universität Hildesheim.

Schweer, Dieter; Seidemann, Sarah (2015): Die neue Macht - digitale Freiräume. In: Sattelberger, Thomas et al. (Hrsg.): Das demokratische Unternehmen. Freiburg u. a.: Haufe Gruppe, S. 129-134.

Welpe, Isabell M.; Tumasjan, Andranik; Theurer, Christian (2015): Der Blick der Managementforschung. In: In: Sattelberger, Thomas et al. (Hrsg.): Das demokratische Unternehmen. Freiburg u. a.: Haufe Gruppe, S. 77-91.

White, Martin (2012): Digital workplaces: Vision and reality. In: Business Information Review 29(4). Sage, S. 205-214. http://jour nals.sagepub.com/doi/pdf/10.1177/0266382112470412

Widén, Gunilla; Steinerová, Jela \& Voisey, Peter (2014): Conceptual modelling of workplace information practices: a literature review. In: Information Research, 19(4). http://www.informa tionr.net/ir/19-4/isic/isic08.html[5.3.2018].

Widén, Gunilla (2017): Individual, social, and cultural approaches to knowledge sharing. In: Journal of Information Science Theory and Practice (JISTaP) vol. 5 http://jistap.org [5.3.2018].

Zheng, Wei; Fang, Hui; Yao Conglei; Wang, Min (2011): Search result diversification for enterprise data. In: Proceedings 20th ACM Conference on Information and Knowledge Management, Glasgow United Kingdom: S. 1901-1904. https://doi.org/ $10.1145 / 2063576.2063850$ [5.3.2018].

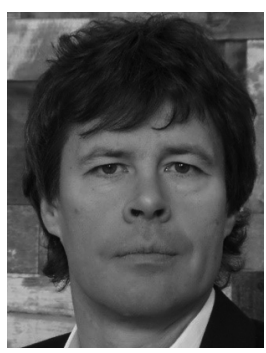

\section{apl. Prof. Dr. Thomas Mandl \\ Universität Hildesheim \\ Institut für Informationswissenschaft und \\ Sprachtechnologie \\ Universitätsplatz 1 \\ 31141 Hildesheim \\ mandl@uni-hildesheim.de \\ www.uni-hildesheim.de/ mandl}

Prof. Dr. Thomas Mandl unterrichtet Informationswissenschaft an der Universität Hildesheim. Seine Forschungsinteressen sind MenschMaschine Interaktion (Usability, Methodenforschung, internationale Aspekte), Digital Humanities und Information Retrieval.

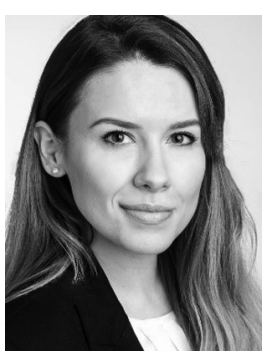

Ella Schwab

Universität Hildesheim

Universitätsplatz 1

31141 Hildesheim

Ella Schwab hat 2017 den Master Internationales Informationsmanagement - Informationswissenschaft an der Universität Hildesheim abgeschlossen. Davor hat sie an der Hochschule Hannover den BA Informationsmanagement erworben.

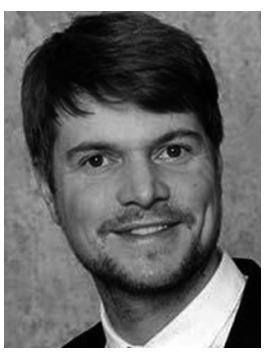

\section{Dr. Ben Heuwing}

Institut für Informationswissenschaft und Sprachtechnologie ]init[ AG für digitale Kommunikation Köpenicker Straße 9 10997 Berlin ben.heuwing@init.de

Ben Heuwing hat an der Universität Hildesheim zu UX und Informationsmanagement geforscht. Aktuell beschäftigt er sich als UX Consultant bei der ]init[ AG insbesondere mit den Themen Digitalisierung, Informationssuche und Kommunikation in Projekten mit Konzernen und für öffentliche Auftraggeber.

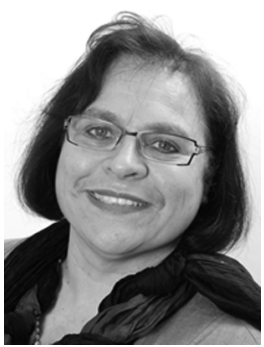

\author{
Prof. Dr. Christa Womser-Hacker \\ Universität Hildesheim \\ Institut für Informationswissenschaft und \\ Sprachtechnologie \\ Universitätsplatz 1 \\ 31141 Hildesheim \\ womser@uni-hildesheim.de \\ www.uni-hildesheim.de/ womser
}

Christa Womser-Hacker ist Professorin für Informationswissenschaft an der Universität Hildesheim. Sie leitet als Direktorin das dortige Institut für Informationswissenschaft und Sprachtechnologie und gehört dem Stiftungsrat der Universität an. Ihre Forschungsschwerpunkte liegen im Bereich des Internationalen Informationsmanagements, insbesondere auf der Mensch-Maschine-Interaktion und dem mehrsprachigen und domänenspezifischen Information Retrieval und dessen Evaluierung. 